\title{
An additional suture secures against pitfalls in delta-shaped gastroduodenostomy after laparoscopic distal gastrectomy
}

\author{
Hirokazu Noshiro $\cdot$ Hironori Iwasaki $\cdot$ Yoshihiro Miyasaka $\cdot$ \\ Kiichirou Kobayashi · Toshihiro Masatsugu • \\ Michiaki Akashi • Osamu Ikeda
}

Received: 9 April 2011/Accepted: 24 July 2011/Published online: 18 August 2011

(C) The International Gastric Cancer Association and The Japanese Gastric Cancer Association 2011

\begin{abstract}
Laparoscopic gastrectomy is widely used as a minimally invasive surgery for gastric cancer. Laparoscopic distal gastrectomy must be followed by either a gastroduodenostomy or gastrojejunostomy to restore continuity of the alimentary tract. The intraabdominal deltashaped gastroduodenostomy using endoscopic linear staplers, which was developed by Kanaya et al., is one of the feasible reconstructive procedures. However, the clinical results still remain uncertain. In 71 patients treated between February 2008 and February 2009, we found that anastomotic failure occurred in six patients and there was an intraabdominal abscess around the anastomosis in two patients, findings which might be associated with technical pitfalls in the procedure. After considering the mechanisms underlying these unfavorable complications, we developed a modification of the procedure to successfully overcome these complications by reinforcement of the anastomosis using simple suturing at the closed common channel on the greater curvature. This modified Kanaya's procedure will be safer and should provide a better intracorporeal gastroduodenostomy after laparoscopic distal gastrectomy.
\end{abstract}

H. Noshiro $(\bowtie) \cdot$ Y. Miyasaka · K. Kobayashi .

T. Masatsugu $\cdot$ M. Akashi · O. Ikeda

Department of Surgery, Faculty of Medicine, Saga University,

5-1-1 Nabeshima, Saga 849-8501, Japan

e-mail: noshiro@cc.saga-u.ac.jp

H. Iwasaki

Department of Surgery, Kyushu Kouseinenkin Hospital,

Kitakyushu, Japan
Keywords Gastric carcinoma - Laparoscopic surgery · Gastroduodenostomy

\section{Introduction}

In the past decade, laparoscopic surgery has been widely accepted as surgical treatment for both benign gastroduodenal diseases and stomach malignancies [1-3]. We and others have reported the advantages of the laparoscopic procedure over the conventional open method [4-6]. Distal gastrectomy must be followed by gastroduodenostomy or gastrojejunostomy to restore continuity of the alimentary tract. The reconstructive procedure in patients with laparoscopic surgery is commonly performed extracorporeally through a minilaparotomy incision and thus is termed laparoscopy-assisted gastrectomy. However, anastomotic procedures that can be performed purely under a laparoscope have been designed for patients with laparoscopic distal gastrectomy (LDG) [7-9]. Some surgeons have reported several intra- or postoperative advantages of totally laparoscopic surgery compared with laparoscopyassisted surgery, although intraabdominal anastomotic procedures are considered to be complex and the endoscopic linear staplers are expensive [10, 11]. A method for Billroth I anastomosis after LDG that uses only endoscopic linear staplers was developed by Kanaya and colleagues in 2002, and has been widely accepted in Asian countries such as Japan and Korea due to its simplicity [7]. However, the clinical results of this procedure have not yet been fully documented. When we reviewed the early surgical outcomes with Kanaya's procedure after LDG at our institutes, to determine the technical pitfalls of the procedure, we found that postoperative complications around the anastomosis had occurred in some patients. In this report, we 
describe a simple method that we contrived to prevent the pitfalls that we noted.

\section{Surgical technique}

The details of the approaches to the intracorporeal deltashaped gastroduodenostomy after LDG are the same as those described by Kanaya et al. [7]. Mobilization and transection of the stomach and lymph node dissection were performed similarly at each institute as described previously [5]. The duodenum was divided in the dorsoventral direction with the use of an endoscopic linear stapler (ETS Flex 45-3.5; Ethicon Endo-Surgery, Cincinnati, OH, USA) immediately after dissection of the infra- and suprapyloric nodes.

\section{Intracorporeal delta-shaped gastroduodenostomy}

A small incision was created on the greater curvature side of the gastric remnant and the posterior side of the duodenum. The 45-mm endoscopic linear stapler was inserted through the left lower port. As the posterior wall of the gastric remnant and that of the duodenum were approximated, the forks of the stapler were closed and fired (Fig. 1a). A V-shaped anastomosis was created on the posterior wall. The common channel through which the stapler had been inserted was closed vertically to the alimentary tract so as to obtain the widest anastomosis, with two additional applications of the endoscopic linear stapler (Fig. 1b). Thereafter, the intracorporeal delta-shaped gastroduodenostomy was made.

\section{Addition of a secure suture to reinforce the anastomosis}

About 1 year after the introduction of Kanaya's procedure, we evaluated the efficacy of the procedure and found that there was a relatively high incidence of complications, such as anastomotic failure and intraabdominal abscess around the anastomosis. At the closed common channel of the greater curvature side, the gastroduodenostomy has the strongest tension and the closing procedure was possibly uncertain because the closing procedure is sometimes hidden between the two forks of the endoscopic linear stapler. Moreover, an extroverted gastroduodenostomy is sometimes attached directly to the bare pancreas after lymph node dissection, and even minimal leakage of pancreatic juice could be activated by attaching the mucosa of the alimentary tract. This may subsequently cause an intraabdominal abscess or leakage of the anastomosis. To investigate these hypotheses and to develop a method to
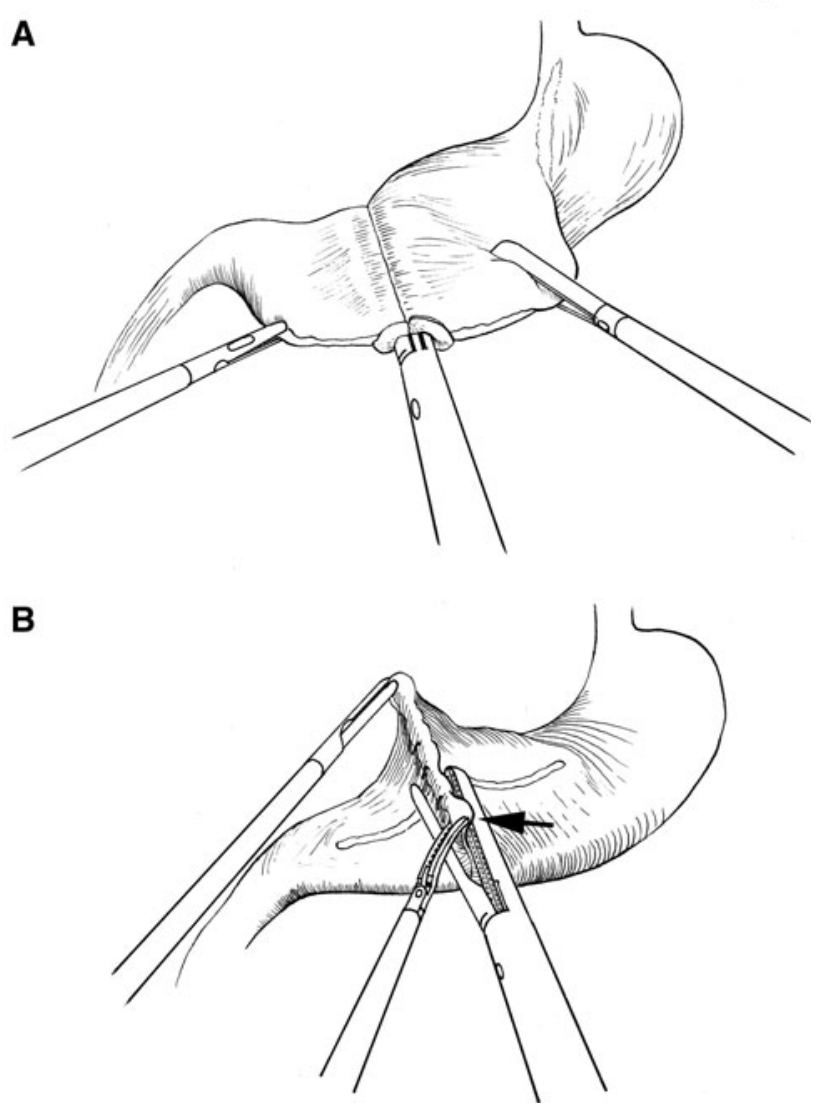

Fig. 1 A schematic representation of the delta-shaped gastroduodenostomy. a The forks of the endoscopic linear stapler are inserted into the small incisions in the duodenal and gastric stumps to create the anastomosis. b The closing process of the small incision using additional endoscopic linear staplers. At the closed common channel of the greater curvature side (arrow), the closing procedure is sometimes hidden between the two forks of the endoscopic linear stapler

avoid the complications, we have reinforced the closed common channel of the greater curvature side by additional suturing with PDSII 4-0 (Fig. 2a, b).

\section{Statistical analysis}

A retrospective analysis was performed, using a prospectively maintained comprehensive database, to determine the technical pitfalls of the procedure. The data from 142 consecutive patients with LDG followed by Kanaya's procedure were collected from our and our affiliate institutions from February 2008 to February 2011. With regard to the clinical data, we obtained the following information from medical charts; age, sex, body mass index (BMI), tumor characteristics, duration of the operation, total estimated blood loss, and complications. The patients who were suspected to have an anastomotic leakage because of pyrexia, leukocytosis, or discharge from a drain (if a drain 

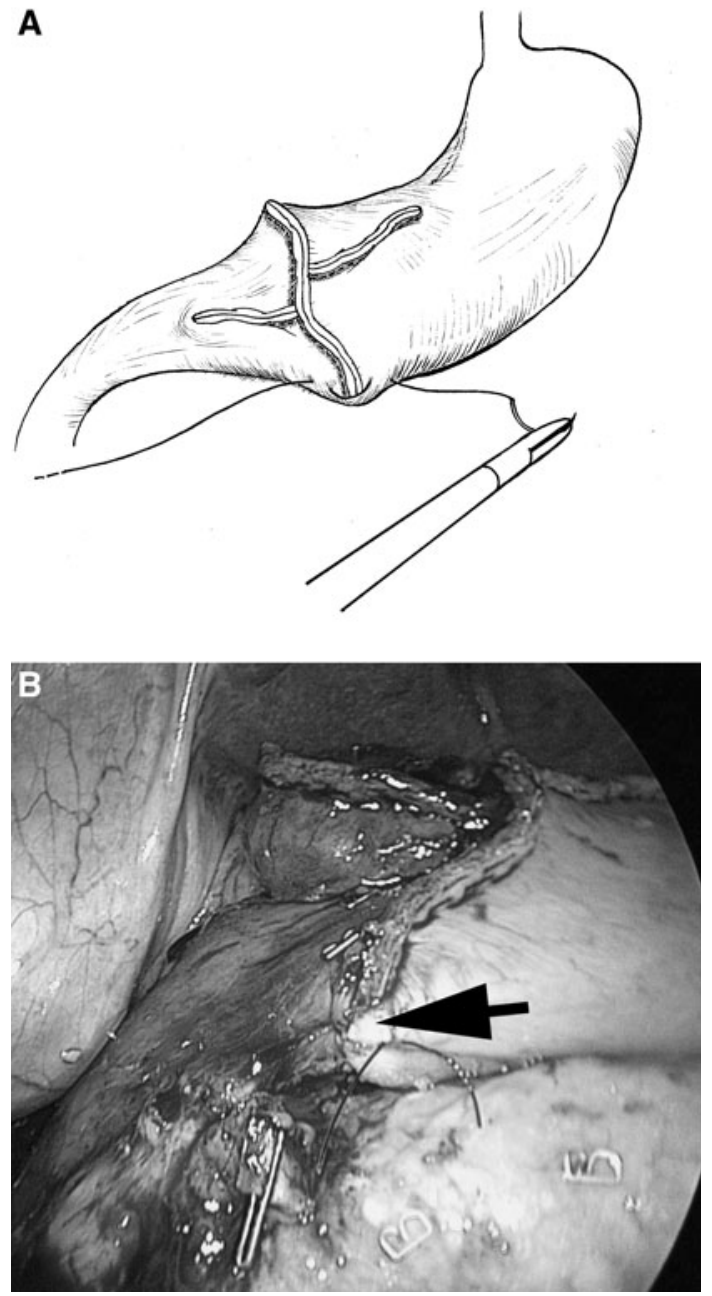

Fig. 2 Additional suturing added to the original Kanaya's procedure to reinforce the anastomosis. a Simple suturing is added on the closed common channel of the greater curvature side. b The extroverted structure in this portion is covered (arrow)

had been placed during surgery) were immediately identified by computed tomography or a gastrograffin swallowing study. Data values are expressed as means \pm standard deviation (SD). Differences between groups were analyzed by Student's $t$-test or the $\chi^{2}$ test using Fisher's exact probability. A two-sided $p$ value of $<0.05$ was considered to be statistically significant.

\section{Results}

Three hundred and two patients with preoperatively diagnosed gastric carcinoma were treated by laparoscopic surgery between February 2008 and February 2011 at our institute and an affiliate institution. Of these patients, 142 underwent an intracorporeal delta-shaped gastroduodenostomy after LDG. The 142 patients could be separated historically into two groups; one group consisted of 71 patients who were treated using the original Kanaya's procedure (Group 1) and the other 71 patients consisted of those who were treated with an additional secure suture that was introduced in March 2009 (Group 2). A summary of the two groups appears in Table 1. There were no significant differences in age, gender, tumor-TNM staging, or extension of lymph node dissection (D1/D2) between the two groups. However, the body mass indices in Group 1 were significantly greater than those in Group 2. The number of patients treated by surgeons with more than 5 years of experience with laparoscopic gastrectomy was similar in the two groups. The duration of the operation was significantly longer for Group 2, but the estimated blood loss did not differ significantly between the two groups. There was no mortality in either group. In the 71 patients in Group 1, there were 6 patients with anastomotic failure and two patients who developed an intraabdominal abscess around the anastomosis after surgery (Table 2). These complications happened in Cases 19, 32, 39, 47, 60, 62,67 , and 71 after the introduction of Kanaya's procedure. The symptoms of leakage occurred within 3 days after surgery with resumption of food intake. As we did not generally place any prophylactic drain in patients with distal gastrectomy, four patients without any drain placed were subjected to reoperation. In two of these patients, the duodenal remnant was somewhat ischemic after its extensive mobilization due to poor mobilization of the gastric remnant, so an information drain was placed around the anastomosis. In these patients we were able to avoid additional surgery due to the effective drainage. The leakage site was found at the caudal closure side of the common channel through which a linear endostapler had been inserted in all patients. Two patients developed delayed intraabdominal abscess around the anastomosis; this happened a week or more after the surgery. In one of these two patients, the abscess was diagnosed on the eleventh postoperative day, after he had been discharged from the hospital. These patients had no symptoms that were suggestive of anastomotic failure immediately after their resumption of food intake. In one of the two patients, roentgenography showed a minute fistula between the abscess and the anastomosis on the greater curvature side. The amylase level in the discharge from the drain was not high, and a bacteria culture study showed no remarkable species of bacteria. There was an anastomotic hemorrhage after surgery in one patient in Group 1. Of nine patients with complications around the anastomosis after surgery, three patients were operated on by a senior surgeon with more than 5 years of experience with laparoscopic gastrectomy and 6 patients were operated on by surgeons with less than 5 years of experience. Since the additional secure suture has been used in combination with the original Kanaya's procedure, there have been no cases of 
Table 1 Clinical characteristics of the patients

\begin{tabular}{llll}
\hline & $\begin{array}{l}\text { Group 1 } \\
n=71\end{array}$ & $\begin{array}{l}\text { Group 2 } \\
n=71\end{array}$ & $p$ \\
\hline Age (years) & $70(10)$ & $67(12)$ & 0.087 \\
Gender (male/female) & $47 / 24$ & $51 / 20$ & 0.592 \\
Body mass index (kg/m $\left.{ }^{2}\right)$ & $22.8(3.1)$ & $21.8(2.8)$ & 0.049 \\
pTNM staging & & & \\
pStage 1 & 57 & 61 & 0.657 \\
pStage 2 & 6 & 4 & \\
pStage 3 & 7 & 6 & \\
pStage 4 & 1 & 0 & \\
Lymph node dissection (D1/D2) & $53 / 18$ & $52 / 19$ & $>0.999$ \\
Duration of operation (min) & $260(56)$ & $305(73)$ & $<0.001$ \\
Blood loss (g) & $63(79)$ & $72(84)$ & 0.579 \\
Surgeon's experience $_{\quad(\text { senior/non-senior) }}{ }^{\mathrm{a}}$ & $30 / 41$ & $36 / 35$ & 0.400 \\
\hline
\end{tabular}

Data are expressed as means (SD). Numbers alone indicate the numbers of patients. Group 1 consisted of the patients treated with Kanaya's original procedure alone between February 2008 and February 2009, and Group 2 consisted of the patients treated with additional suturing and Kanaya's procedure between March 2009 and February 2011

${ }^{a}$ A senior surgeon was defined as one who had more than 5 years of experience performing laparoscopic gastrectomies

Table 2 Postoperative complications

\begin{tabular}{lcll}
\hline & $\begin{array}{l}\text { Group 1 } \\
n=71\end{array}$ & $\begin{array}{l}\text { Group 2 } \\
n=71\end{array}$ & $p$ \\
\hline $\begin{array}{l}\text { Complications around the anastomosis } \\
\quad \text { Anastomotic failure }\end{array}$ & 6 & 0 & 0.028 \\
$\begin{array}{l}\text { Intraabdominal abscess } \\
\text { around the anastomosis }\end{array}$ & 2 & 0 & 0.497 \\
Anastomotic hemorrhage & 1 & 0 & $>0.999$ \\
$\quad$ Anastomotic stricture & 0 & 0 & $>0.999$ \\
Other abdominal complications & 4 & 3 & $>0.999$ \\
Respiratory complications & 2 & 1 & $>0.999$ \\
Total & 15 & 4 & 0.012 \\
\hline
\end{tabular}

anastomotic leakage or intraabdominal abscess around the anastomosis thus far. No stricture of the anastomosis was observed in either group. The incidences of other complications were similar in the two groups (Table 2).

\section{Discussion}

Recently, surgical techniques and modalities have advanced to allow adequate dissection of regional lymph nodes and safe reconstruction during laparoscopic surgery for the treatment of gastric carcinoma. However, there are still problems with anastomotic procedures after laparoscopic gastrectomy that must be addressed. Gastroduodenostomy, the so-called Billroth I reconstruction, is most commonly performed after LDG to facilitate anastomosis. The laparoscopic gastroduodenostomy procedure without the use of a circular stapler was developed by Kanaya, and the procedure has been employed at our institute since February of 2008.

Conventionally, a gastroduodenostomy can be performed using a circular stapler through a minilaparotomy [2], but this is sometimes difficult when the gastric or duodenal remnant is too small to be handled through the minilaparotomy. Docking the anvil head with the center rod of a circular stapler through the minilaparotomy is not easily performed, and sometimes may result in injuries to the structures around the anastomosis, due to forceful tension, or may result in involvement of the surrounding tissues. Moreover, intracorporeal anastomosis requires a smaller incision of about 3-cm for removing the excised specimen, compared with an extended incision of at least $5-\mathrm{cm}$ at the epigastric lesion in an extracorporeal anastomosis. This suggests that totally laparoscopic surgery might be less traumatic and less invasive.

Kanaya was the first surgeon who reported a purely laparoscopic method for Billroth I anastomosis after LDG that uses only endoscopic linear staplers [7]. Kanaya's procedure has been widely accepted, especially in Japan and Korea, due to its simplicity. Kanaya and his colleagues reported the safety and feasibility of the procedure with their experience of 100 patients [12]. In their series, the rate of anastomotic failure was only $1 \%$. However, there are few reports by other institutes that have followed Kanaya's procedure. The clinical results of the original Kanaya's procedure have not yet been fully documented.

We herein reviewed the early surgical outcomes of this procedure at our institutions. There were six patients with anastomotic failure and two patients with intraabdominal abscess around the anastomosis in the 71 patients treated with the original Kanaya's procedure the first year after the introduction of this type of reconstruction. These were serious problems with relatively high incidences, and the benefits associated with less invasive surgery might be decreased by such complications. These problems could not be explained by technical immaturity alone because we spent much time, including practice surgeries in an animal laboratory, to become skilled in this procedure, and we were instructed directly by either Kanaya or his colleagues. Moreover, these postoperative complications were not only associated with young surgeons but also with experienced surgeons, and these adverse events did not happen only during the early phase just after the introduction of the procedure. In addition, the BMI of the patients in this study could not account for the present complications because the 
approximately $1.0-\mathrm{kg} / \mathrm{m}^{2}$ difference in the mean BMIs between the two groups was too small to have a serious impact on the postoperative complications. The reason for the significantly shorter duration of operation in the earlier group was unclear, but it was unlikely to be associated directly with the problem with the anastomosis.

Therefore, we speculated on possible reasons for these complications, and contrived methods for preventing complications involving the anastomosis. First, we noted that the gastroduodenostomy had the strongest tension at the caudal side of the closed common channel of the greater curvature. Nevertheless, the closing procedure was uncertain at this portion because this portion tends to roll backward during the procedure of closing the common channel behind the inserted linear endostapler. By reoperation or roentgenography, we confirmed that this portion was associated with the anastomotic failure. Next, we observed that the extroverted gastroduodenostomy was sometimes attached directly to the bare pancreas after radical lymph node dissection, and even minimal leakage of pancreatic juice could be activated by the attachment of mucosa of the alimentary tract. Pancreatic juice is activated by intestinal factors or bacteria and digests the surrounding tissues that are exposed to the activated enzymes. Obama et al. [13] showed that leakage of pancreatic juice was more common in laparoscopic gastrectomy than in conventional open gastrectomy. Consequently, intraabdominal abscess or leakage of the anastomosis might be caused by the pancreas head after radical lymph node dissection. We believed that the development of a serious pancreatic fistula caused by radical lymph node dissection was not likely, because the level of amylase in the purulent discharge from the abscess was not highly elevated.

To confirm these hypotheses and to avoid the unfavorable complications observed during the early surgical outcomes, we started reinforcing the closed common channel of the greater curvature side and we covered the extroverted anastomosis by simple additional suturing. Consequently, complications such as anastomotic leakage and intraabdominal abscess around the anastomosis have disappeared. This favorable result appears to support our hypotheses.

We conclude that the addition of simple suturing to secure the anastomosis with Kanaya's procedure can provide a safer and more feasible intracorporeal gastroduodenostomy after LDG.

Acknowledgments The authors are grateful for the technical training and ongoing advice on the operative procedures provided by Dr. Seiichiro Kanaya at the department of surgery of Fujita Health University School of Medicine.
Conflict of interest Drs. Hirokazu Noshiro, Tomonori Iwasaki, Yoshihiro Miyasaka, Kiichirou Kobayashi, Toshihiro Masatsugu, Michiaki Akashi, and Osamu Ikeda have no conflicts of interest or financial ties to disclose.

\section{References}

1. Shimizu S, Noshiro H, Nagai E, Uchiyama A, Tanaka M. Laparoscopic gastric surgery in a Japanese institution: analysis of the initial 100 procedures. J Am Coll Surg. 2003;197:372-8.

2. Kitano S, Shiraishi N, Uyama I, Tanigawa N. Japanese Laparoscopic Surgical Study Group A multicenter study on oncologic outcome of laparoscopic gastrectomy for early cancer in Japan. Ann Surg. 2007;245:68-72.

3. Kitano S, Shiraishi N. Current status of laparoscopic gastrectomy for cancer in Japan. Surg Endosc. 2004;18:182-5.

4. Shimizu S, Uchiyama A, Mizumoto K, Morisaki T, Nakamura K, Shimura H, Tanaka M. Laparoscopically assisted distal gastrectomy for early gastric cancer: is it superior to open surgery? Surg Endosc. 2000;14:27-31.

5. Noshiro H, Nagai E, Shimizu S, Uchiyama A, Tanaka M. Laparoscopically assisted distal gastrectomy with standard radical lymph node dissection for gastric cancer. Surg Endosc. 2005;19:1592-6.

6. Adachi Y, Shiraishi N, Shiromizu A, Bandoh T, Aramaki M, Kitano S. Laparoscopy-assisted Billroth I gastrectomy compared with conventional open gastrectomy. Arch Surg. 2000;135: 806-10.

7. Kanaya S, Gomi T, Momoi H, Tamaki N, Isobe H, Katayama T, Wada Y, Ohtoshi M. Delta-shaped anastomosis in totally laparoscopic Billroth I gastrectomy: new technique of intraabdominal gastroduodenostomy. J Am Coll Surg. 2002;195:284-7.

8. Kim JJ, Song KY, Chin HM, Kim W, Jeon HM, Park CH, Park SM. Totally laparoscopic gastrectomy with various types of intracorporeal anastomosis using laparoscopic linear staplers: preliminary experience. Surg Endosc. 2008;22:436-42.

9. Tanimura S, Higashino M, Fukunaga Y, Takemura M, Nishikawa T, Tanaka Y, Fujiwara Y, Osugi H. Intracorporeal Billroth I reconstruction by triangulating stapling technique after laparoscopic distal gastrectomy for gastric cancer. Surg Laparosc Endosc Percutan Tech. 2008;18:54-8.

10. Song KY, Park CH, Kang HC, Kim JJ, Park SM, Jun KH, Chin $\mathrm{HM}$, Hur H. Is totally laparoscopic gastrectomy less invasive than laparoscopy-assisted gastrectomy? Prospective, multicenter study. J Gastrointest Surg. 2008;12:1015-21.

11. Kim MG, Kawada H, Kim BS, Kim TH, Kim KC, Yook JH, Kim BS. A totally laparoscopic distal gastrectomy with gastroduodenostomy (TLDG) for improvement of the early surgical outcomes in high BMI patients. Surg Endosc. 2010;25:1076-82.

12. Kanaya S, Kawamura Y, Kawada H, Iwasaki H, Gomi T, Satoh S, Uyama I. The delta-shaped anastomosis in laparoscopic distal gastrectomy: analysis of the initial 100 consecutive procedures of intracorporeal gastroduodenostomy. Gastric Cancer. doi:10.1007/ s10120-011-0054-0.

13. Obama K, Okabe H, Hosogi H, Tanaka E, Itami A, Sakai Y. Feasibility of laparoscopic gastrectomy with radical lymph node dissection for gastric cancer: from a viewpoint of pancreas-related complications. Surgery. 2011;149:15-21. 